\title{
Comparison between Kangaroo Mother Care with Standard Care in Preterm Neonate Management
}

\author{
${ }^{*}$ Chowdhury $\mathrm{RM}^{1}$, Shahidullah $\mathrm{M}^{2}$, Mannan $\mathrm{MA}^{3}$, Chowdhury MAA ${ }^{4}$, Biswas $\mathrm{BC}^{5}$, Das $\mathrm{KP}^{6}$
}

\begin{abstract}
Preterm low birth weight (LBW) babies are unable to control their body temperature and are at greater risk of illness. Kangaroo Mother Care (KMC) is special ways of caring for low birth weight babies. KMC provide thermal care through continuous skin to skin contact, support for exclusive breast feeding and early recognition and response to complication. The aim of the study is to assess thermal control, morbidities feeding pattern of baby getting KMC in comparison to conventional care. The study also give estimation about duration and cost during hospital stay and growth between the babies of two groups. This Randomized Controlled Trial was conducted in the Department of Neonatology, BSMMU, Dhaka from December 2012 to October 2013. Fifty neonate with birth weight 1250 to $1800 \mathrm{gm}$, gestational age >30 weeks to $<37$ weeks, Hemodynamically stable after birth were selected. Twenty five of them were randomly allocated to KMC(Group-I) and 25 of them to Standard Method Care (Group-II). The mother or care giver provided KMC at least 12 hours/day in Group-I. Those in Group-II were managed under radiant warmer. During hospital stay both the groups were monitored and after discharge, the neonates were followed weekly till 40 weeks of corrected gestational age. There were no differences in birth weight, gestational age, number of malelfemale neonates, places of delivery and mode of delivery between two groups. During hospital stay hypothermia
\end{abstract}

1. *Dr. Rumpa Mani Chowdhury, Junior consultant, NICU, Green Life Hospital, Green Road, Dhaka. Mobile: 01816356807, E-mail: rumpamonichowdhury@gmail.com

2. Dr. Mohammod Sahidullah, Professor \& Chairman, Department of Neonatology, BSMMU.

3. Dr. Md. Abdul Mannan, Professor, Department of Neonatology, BSMMU.

4. Dr. M.A. Azad Chowdhury, Prof. \& Head, Department of Neonatology, Bangladesh Institute of Child Health \& Dhaka Shishu Hospital, Dhaka

5. Dr. Bidhan Chandra Biswas, Assistant professor, Department of Neonatology, Sher e Bangla Medical C ollege, Barishal

6. Dr. Krishna Priya Das, Associate professor, Department of Orthopaedics, BSMMU, Dhaka

${ }^{*}$ For Correspondence
(Group-I 4\% vs Group-II 24\%) and hyperthermia (Group I $8 \%$ vs Group II 32\%) were significantly low in KMC group. Late onset neonatal sepsis (LONS) developed significantly in Group-II. Though incidence of culture negative LONS did not differ in two groups but incidence of blood culture positive LONS was significantly high in standard care neonates $36 \%$ vs.0\%; p=0.001). Neonates with KMC care required significantly shorter time to reach full feeding and to start direct breast feeding; also incidence of feeding intolerance was significantly lower in this group. After initial loss, weight gain started earlier as well as achieved the birth weight earlier in KMC group. Morbidities like hyperglycaemia and apnoea were significantly higher in standard care group. Neonates who received standard care stayed significantly longer in the hospital and total cost during hospital stay was also significantly higher in this group. At 40 weeks corrected gestational age, KMC infants showed significantly higher daily weight gain than standard care group. This study concluded that Kangaroo Mother Care provides effective thermal control, decreases the incidence of sepsis, improves feed tolerance, helps to achieving full enteral feeding and birth weight earlier in preterm $L B W$ neonates. KMC enhances growth during postnatal period. KMC also found to reduce hospital stay and treatment cost. Proper implementation of KMC for the management of preterm low birth weight babies is safe and effective care.

Keywords: Kangaroo mother care, Low birth weight, Breastfeeding, Postnatal growth.

\section{INTRODUCTION}

Low birth weight babies have an adverse effect on child survival and development. World-wide, twenty-five millions LBW infants are born each year, most of them (96\%) in developing countries. Preterm birth is the single most important cause of neonatal mortality, accounting for an estimated $27 \%$ of the 4 million neonatal deaths every year. ${ }^{1}$ Worldwide each year at least 15 million babies are born too soon each year before 37 completed weeks of pregnancy that is one out of every 10 babies. $^{2}$ In Bangladesh total 421, 200 babies had born preterm LBW. ${ }^{3}$ Preterm LBW infants are predisposed to heat loss because they have a high ratio of surface area to body weight, little subcutaneous fat, and reduced glycogen and brown 'fat' 
stores. In addition, their hypotonic ("frog") posture limits their ability to curl up to reduce the skin area exposed to cold environment. ${ }^{4}$ Conventionally these babies are managed under radiant warmer or incubator which is expensive and needs both trained personnel and permanent logistic support. The baby under warmer has increased risk of temperature instability, increase chance of nosocomial infection and increase insensible loss of water. ${ }^{4}$

In 1978 Edger Rey, a Colombian pediatrician first described Kangaroo Mother Care; 5 a neonatal care technique for low birth weight infants is as effective as traditional care. ${ }^{6}$ In KMC caring the preterm or low birth weight infants are kept in skin-to-skin contact with the mother and breastfed exclusively. It is also known as kangaroo care $(\mathrm{KC})$ because it can be provided by personnel other than mother like father, grandmother or any other member of family with ensuring exclusive breastfeeding. The major components of KMC are (1) Skin-to-skin contact. The babies are kept firmly against in between mother's breasts in an upright position (2) ensured exclusive breast feeding (3) early discharge from hospital regardless of weight or gestational age. ${ }^{7}$ The reported advantages of KMC for the infant are well maintained of skin temperature, reduce incidence of apnea and bradycardia, stable transcutaneous oxygen level, longer quiet sleep periods, shorter hospital stay and therefore have potential for considerable cost savings. ${ }^{8}$ KMC decreases the incidence of nosocomial infection, reduce severe morbidity, avoid hospital readmission and increase weight gain in comparison to conventional care group. ${ }^{9,10} \mathrm{KMC}$ has also been found higher breast feeding rate and improvement of maternal confidence and better bonding with their infant. ${ }^{11}$

\section{Rationale of the study:}

Among the three major causes of all newborn deaths, death due to preterm related complication account $45 \% .{ }^{12}$ Bangladesh has already achieved the MDG 4 and on the way to achieve SDG. So to achieving this goal neonatal death should be reduced. For the management of LBW babies by standard conventional method is a big burden for the society and nation. If we establish KMC in our country LBW baby management will become convenient for us. This study will give us a decision about the feasibility of $\mathrm{KMC}$ in our country and it will help us to overcome the burden of expense for the management of preterm LBW babies.

Objective: The present study attempted to find thermal control, morbidities, exclusive breast feeding rate, duration and cost during hospital stay and growth between the babies of two groups.

\section{MATERIALS AND METHODS}

This randomized controlled trial was conducted in the Department of Neonatology, BSMMU, Dhaka over a 11 months period. Total 50 neonates were studied who were admitted in NICU according to following inclusion and exclusion criteria.

\section{Inclusion criteria:}

-Neonate with birth weight 1250 to $1800 \mathrm{gm}$.

-Gestational age $>30$ weeks to $<37$ weeks

- Hemodynamic ally stable after birth.

\section{Exclusion criteria:}

-Major life threatening congenital malformation,

- Severe perinatal asphyxia,

- Babies require ventilator or inotropic support,

- Mother is critically ill or unable to comply with the follow up schedule

- Parental refusal for KMC intervention.

The subjects were divided into two groups; Kangaroo Mother Care (Group-I) and Standard Method Care (Group-II). Randomization was achieved by lottery technique and allocation was concealed by sealed envelope technique.

In the KMC group mothers were explained detail about $\mathrm{KMC}$ adoption. $\mathrm{KMC}$ was initiated as soon as the baby was stable. If the mother is not available initially any of the family members can start KMC. The mother provided skin to skin contact in upright position dressed with a cap, socks and diaper and supported in bottom with a sling/binder. Adequate privacy was ensured. Comfortable chairs and beds were provided to the mothers practicing $\mathrm{KMC}$ in the nursery. Skin-to skin contact was given for a minimum of 1 hour at a time and at least for 12 hrs./ day, duration was gradually increased to as long as comfortable to the mother and baby. When the baby is not in KMC, the baby was placed in the cot with adequately clothed and covered. Neonate in the SMC group was managed under radiant warmer. In both the groups, mothers were allowed to handle their babies at any hour of the day breastfeed the babies by nasogastric tube, cup-spoon or directly. Babies in 
both the groups were provided feeding, vitamin and minerals supplementation as per protocol.

During hospital stay, both the groups were monitored for daily weight gain by electronic weighing scale, episodes of hypothermia, apnea, feeding intolerance, nosocomial sepsis, hyperbilirubinemia, necrotizing enter colitis, physiological parameters (heart rate, respiratory rate, axillary temperature and oxygen saturation) were measured by a single observer and duration of stay at hospital was recorded. Weight gain pattern, time to reach full enteral feeding, adequacy of breast feeding and duration of hospital stay was also recorded.

Neonates in both the groups were discharged when baby's general health is good, no evidence of infection and no I/V

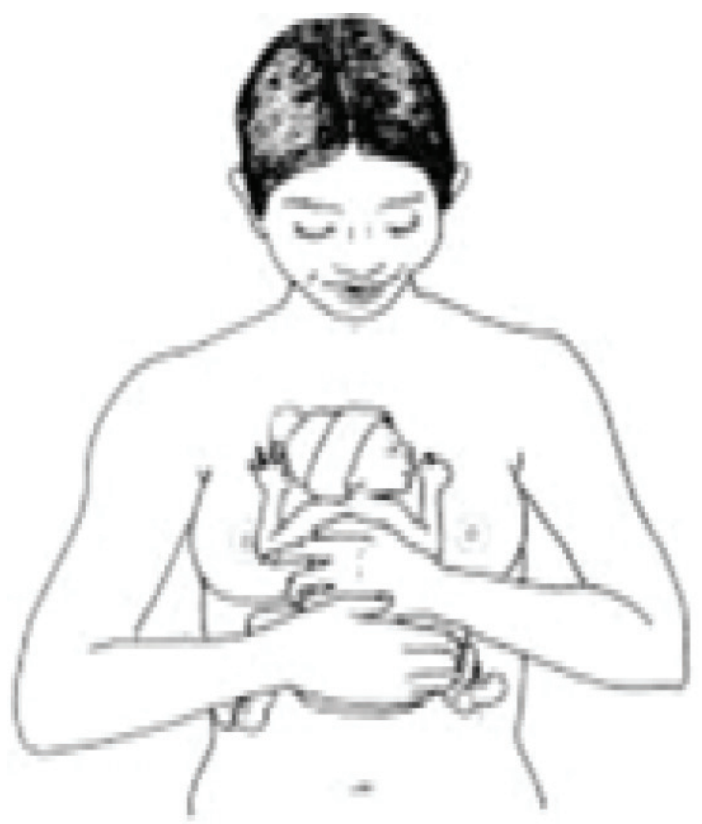

Fig-1: Kangaroo Position of baby and mother

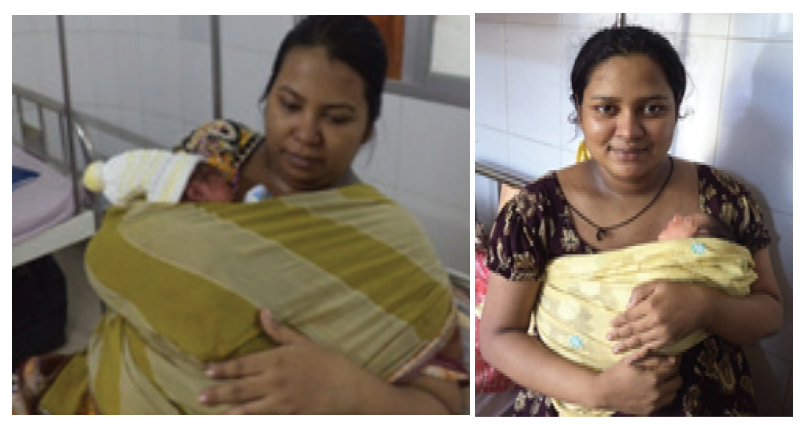

Fig-2\&3: Mother giving KMC in recumbent position and sitting position medications, baby is feeding well and receiving breast milk directly or by cup-spoon, gaining weight (at least 15-20 $\mathrm{gm} . / \mathrm{kg} /$ day) for three consecutive day, maintaining body temperature satisfactorily without assistance and mother and family members are confident to care of the baby. After discharge, the neonate was followed weekly till 40 wks. of gestational age in preterm babies. Data were recorded in the follow up sheet. The approval from the Institutional review board was obtained prior to the study. An informed written consent was taken from the mothers or care givers.

\section{STATISTICAL ANALYSIS}

Data were collected by standard questionnaire and check list, compiled and analyzed with the help of SPSS version 20.0. Quantitative data were expressed as mean and standard deviation and comparison will be done by unpaired student's' test.Qualitative data were expressed as frequency and percentage and comparison, carried by chi-square $\left(\mathrm{X}^{2}\right)$ test. Probability value $(\mathrm{p})$ of less than 0.05 was considered as statistical significance.

\section{RESULTS}

The baseline characteristics were compared in Table-I, significant differences were found in between two groups. In table-II, hypothermia, hyperthermia, and sepsis were found significantly higher in SMC group compared to the KMC group. Episode of apnea found significantly lower in KMC group. Incidence of feeding intolerance differed significantly in two groups (Group I $8 \%$ vs. Group II 56\%; $\mathrm{p}=0.001$ ). Neonates with KMC care required significantly shorter time to reach full feeding (Group I 8.32 \pm 2.49 days vs. Group II $19.56 \pm$ 6.80 days; $\mathrm{p}=0.002)$. After initial loss, weight gain started earlier (Group- I 9.92 \pm 1.55 days vs. Group II $14.96 \pm 2.31$ days; $\mathrm{p}=0.001)$ as well as achieved the birth weight earlier (Group I 16.56 6.29 days vs. Group II $22.76 \pm 7.20$ days; $\mathrm{p}=0.001)$ in $\mathrm{KMC}$ group. Neonates with KMC care required statistically significant shorter duration to start direct breastfeeding than standard care group (Group I 9.6 2.16 days vs. Group II $20.12 \pm 3.82$ days; $\mathrm{p}=0.04$ ). (Table -III). At corrected 40 weeks of gestational age, KMC infants showed significantly higher daily weight gain than standard care group. 
Table-I Neonatal baseline characteristics

\begin{tabular}{|c|c|c|c|}
\hline Variables & $\mathrm{KMC}(\mathrm{n}-25)$ & SMC (n-25) & $\mathrm{p}$ value \\
\hline Gestational age(wks.; mean \pm SD) & $32.96 \pm 1.27$ & $33.16 \pm 1.40$ & 0.49 \\
\hline Birth weight(gms.;mean \pm SD) & $1566 \pm 155.94$ & $1563 \pm 146.13$ & 0.38 \\
\hline \multicolumn{4}{|l|}{ Male: Female ratio } \\
\hline Mode of delivery n (\%) & $1.77: 1$ & $0.66: 1$ & 0.08 \\
\hline NVD & $5(20 \%)$ & $7(28 \%)$ & \\
\hline LUCS & $20(80 \%)$ & $17(68 \%)$ & \\
\hline Assisted delivery & $0(0 \%)$ & $4(1 \%)$ & \\
\hline \multicolumn{4}{|l|}{ Birth weight groups n (\%) } \\
\hline 1250-1400gms. & $4(16 \%)$ & $5(20 \%)$ & \\
\hline $1401-1600 \mathrm{gms}$ & $8(32 \%)$ & $8(32 \%)$ & 0.92 \\
\hline $1601-1800 \mathrm{gms}$ & $13(52 \%)$ & $12(48 \%)$ & \\
\hline \multicolumn{4}{|l|}{ Gestational age groups n (\%) } \\
\hline 30-32 weeks & $12(48 \%)$ & $9(36 \%)$ & \\
\hline 33-34 weeks & $9(36 \%)$ & $11(44 \%)$ & 0.69 \\
\hline $35-<37$ weeks & $4(16 \%)$ & $5(20 \%)$ & \\
\hline \multicolumn{4}{|c|}{ Classification based on Lubchenco's charts n (\%) } \\
\hline Appropriate for gestational age (AGA) & $23(92 \%)$ & $18(72 \%)$ & 0.06 \\
\hline Small for gestational age (SGA) & $2(8 \%)$ & $7(28 \%)$ & \\
\hline
\end{tabular}

KMC- Kangaroo Mother Care; SMC- Standard Mother Care.

Table-II: Effect of KMC on morbidities

\begin{tabular}{|l|c|c|c|}
\hline Variables & KMC(n-25) & SMC(n-25) & P Value \\
\hline Hypothermia n (\%) & $1(4 \%)$ & $6(24 \%)$ & 0.04 \\
\hline Hyperthermia n (\%) & $2(8 \%)$ & $8(32 \%)$ & 0.03 \\
\hline Late onset neonatal sepsis n (\%) & $09(36 \%)$ & $16(64 \%)$ & 0.04 \\
\hline Culture negative sepsis n (\%) & $0(0 \%)$ & $6(24 \%)$ & 0.009 \\
\hline Hypoglycaemia n (\%) & $1(4 \%)$ & $5(20 \%)$ & 0.08 \\
\hline Hyperglycaermia n (\%) & $1(4 \%)$ & $6(24 \%)$ & 0.04 \\
\hline Apnea n (\%) & $2(8 \%)$ & $8(32 \%)$ & 0.03 \\
\hline Neonatal Jaundice n(\%) & $16(64 \%)$ & $19(76 \%)$ & 0.35 \\
\hline Hospital stay(days; mean \pm SD & $12.04 \pm 2.74$ & $25.24 \pm 7.20$ & 0.04 \\
\hline
\end{tabular}

KMC- Kangaroo mother care; SMC- Standard method care. 
Table-III: Effect on feeding and weight gain pattern in two groups

\begin{tabular}{|l|c|c|c|}
\hline Variables & KMC(n-25) & SMC(n-25) & P Value \\
\hline Time of starting breast feeding (days) (mean \pm SD) & $9.6 \pm 2.16$ & $20.12 \pm 3.82$ & 0.04 \\
Feeding intolerance $\mathrm{n}(\%)$ & $2(8 \%)$ & $14(56 \%)$ & 0.001 \\
Start of feed(days) (mean $\pm \mathrm{SD})$ & $1.80 \pm 0.40$ & $2.20 \pm 0.40$ & 0.1 \\
\hline Days to rech full enteral feed(days) (mean \pm SD) & $8.32 \pm 2.49$ & $19.56 \pm 6.80$ & 0.002 \\
\hline Adequacy of breast milk production $\mathrm{n}(\%)$ & $22(88 \%)$ & $16(64 \%)$ & 0.04 \\
\hline Exclusive breast feeding rate upto follow up $\mathrm{n}(\%)$ & $25(100 \%)$ & $23(92 \%)$ & 0.14 \\
\hline Weight gain started(days) (mean $\pm \mathrm{SD})$ & $9.92 \pm 1.55$ & $14.96 \pm 2.31$ & 0.001 \\
\hline Birth weight Regain(days)( mean $\pm \mathrm{SD})$ & $16.56 \pm 6.29$ & $22.76 \pm 7.20$ & 0.001 \\
\hline
\end{tabular}

KMC- Kangaroo mother care; SMC- Standard method care.

Table-IV: Effect of growth in two groups at $\mathbf{4 0}$ weeks corrected gestational age

\begin{tabular}{|l|c|c|c|}
\hline Variables & $\begin{array}{c}\text { Group-I }(\mathrm{n}=25) \\
\text { mean } \pm \text { SD }\end{array}$ & $\begin{array}{c}\text { Group-II }(\mathrm{n}=25) \\
\text { mean } \pm \text { SD }\end{array}$ & p value \\
\hline Weight gain (gm./day) & $27.08 \pm 3.02$ & $16.00 \pm 2.76$ & 0.002 \\
\hline Head circumference gain (cm/wk.) & $0.86 \pm 0.09$ & $0.81 \pm 0.09$ & 0.07 \\
\hline Length gain (cm/wk.) & $0.99 \pm 0.16$ & $0.92 \pm 0.10$ & 0.08 \\
\hline
\end{tabular}

\section{DISCUSSION}

Kangaroo mother care has been proposed as an alternative method for caring preterm low birth weight babies. Neonates in both the groups were found to be comparable in regard to gestational age and birth weight between the groups. Most of the patients were appropriate for gestational age (AGA).Similarly RCT, conducted on comparing KMC and conventional care reported almost identical proportion of AGA. ${ }^{7}$ Another study recruited preterm LBW neonates who were SGA. ${ }^{13}$ Most of the neonates were between 1601-1800 grams weight range in both the groups (KMC: $52 \%$ and standard care $48 \%$ ) and the difference was not statistically significant. Similar weight distribution $(58 \%$ in $\mathrm{KMC}$ and $50 \%$ in conventional care) was observed in other related study. ${ }^{7}$

During the hospital stay, higher incidence of hypothermia ( $4 \%$ vs. $24 \%$ ) occurred in KMC group and Standard care group respectively, which was statistically significant. This finding was comparable with another study. ${ }^{13}$ Hypothermia was found significantly lower in KMC group ( $6 \%$ vs.37\%, $\mathrm{p}=<0.001)$. Episodes of hypothermia were significantly reduced in KMC group and higher rectal temperature was recorded. ${ }^{7}, 14$ Same results also found in other studies. ${ }^{15,16,17}$ In another RCT states that hypothermia was significantly less common (13.5 vs. 31.5 episodes/100 infants) in KMC infants. ${ }^{18}$ Incidence of hyperthermia was also found higher and statistically significant in standard care group in this study (32\% vs. $8 \%$ ). Similarly higher incidence of hyperthermia was also found in another study. ${ }^{13}$

A large proportion (64\%) of neonates in standard care group developed sepsis during the study period. In another prospective RCT on 114 preterm LBW neonates, statistically significant sepsis was found in conventional care group that was $23.2 \%$ vs. $6.9 \% \quad(p=0.014) .{ }^{7}$ Significantly low incidence of sepsis was also reported by other study. ${ }^{12}$ Among 206 LBW babies significantly lower incidence of sepsis was found in $\mathrm{KMC}$ group $3.9 \%$ vs. $14.8 \% \mathrm{p}=0.008$. Cochrane review concluded that KMC reduces the incidence of nosocomial infections. ${ }^{19}$ In a prospective observational study also found low incidence of sepsis $(4.7 \%) .^{20}$

There were 6 culture positive sepssis and 10 culture negative sepsis in conventional care group (Group-II). On 
the other hand there was no culture positive sepsis in KMC group though 9 neonates diagnosed as culture negative sepsis were found. At that time there were so many culture positive cases in the ward. Most probably for this reason, culture positive sepsis was more prevalent in conventional care group.KMC decreases the incidence of nosocomial infection; this benefit is more significant in developing rather than developed countries. ${ }^{21}$

This study showed, significantly higher number of neonates in the standard care group suffered from hyperglycemia ( $4 \%$ vs. $24 \%$ ) compared to KMC group. Higher numbers of babies suffered from sepsis in standard care group which causes glucose intolerance. Hyperglycemia may be found higher due to sepsis in SMC group. Hypoglycemia was found higher percentage in standard care group ( $20 \%$ vs. $4 \%$ ) in this study. Similar findings also found in another study. ${ }^{13}$ In our study KMC was found significantly reduce the incidence of apnea (32\% vs. $8 \%$ ) that is similar to other study. ${ }^{7,} 21 \& 22$ As regards to hospital stay kangaroo mother care reduced hospital stay significantly $(p<0.05)$ in KMC group than standard method care group $(12.04 \pm 2.74$ vs. 25.24 \pm 7.20 ). Early attainment of full enteral feed and shorter hospital stay possibly fewer infection episodes in KMC group that is comparable with other study. ${ }^{7,13,22}$ In another study, neonates in the KMC group were discharged earlier from hospital ( $27.2+/-7$ vs. $34.6+/-7$ days) than standard care group. ${ }^{23}$

This study showed that mean (days) $\pm \mathrm{SD}$ to reach full enteral feed was $8.32 \pm 2.49$ and $19.56 \pm 6.80$ in KMC group and standard care group respectively and this difference was statistically significant. In another prospective RCT it was shown that less time required toreaching full enteral feeding in $\mathrm{KMC}$ group. This study showed that neonates who got KMC required statistically significant shorter time to start breastfeeding directly than standard care group. Another study also showed same type of results. ${ }^{12}$ Adequate amount of breast milk was significantly higher in KMC group mother in this study. Adequacy of breast milk was assessed by daily urine output and weight gain of baby. KMC promoted longer duration of breastfeeding, higher volumes of milk production, exclusive breastfeeding rates at the time of discharged from hospital that revealed with other study. ${ }^{24,25}$ Studies carried out in areas where $\mathrm{KMC}$ is done show that mothers who establish skin to skin contact with their preterm babies have a significantly higher milk production than their control group. Furthermore these studies have also revealed that interruption of breastfeeding was more frequent among mothers who were not submitted to this method. ${ }^{26,27,28}$ We always try to ensure breast milk for the baby from mother or donor milk, formula feeding is not recommended in our institution. Exclusive breastfeeding rate was higher in KMC group $(100 \%$ vs. $92 \%)$ but was not statistically significant. A randomized trial carried out with KMC among babies less than 1500 gram which found higher breastfeeding rates $(55 \%$ vs. $28 \%)$ at 6 weeks in $\mathrm{KMC}$ group. ${ }^{29}$ The number of mothers exclusively breastfeed their babies at 6 week follow-up was double (12/14 vs. 6/14) in the KMC group than in the control group. ${ }^{30}$ Another comparative study also found higher rate of exclusive breastfeeding in KMC group. ${ }^{13} \mathrm{KMC}$ was found to decrease probability of not exclusively breastfeeding (relative risk $0.41,95 \%$ confidence interval 0.25 to 0.68 ) at discharge. ${ }^{31}$ (KMC: $94.4 \%$, control: $72 \%, p=0.002$ ).

In this study, KMC affect both start of weight gain and birth weight regain significantly. In this study at 40 weeks corrected gestational age, KMC neonates showed significantly higher daily weight gain than standard care group (Group I 27.08 3.02 gms vs. Group II 16.00 \pm 2.76 gms; $\mathrm{p}=0.002)$, head circumference gain $(\mathrm{cm} / \mathrm{wk}$.) $0.86 \pm$ 0.09 and $0.81 \pm 0.09$ and length gain $(\mathrm{cm} /$ wk.) was $0.99 \pm$ 0.16 and $0.92 \pm 0.10$ in $\mathrm{KMC}$ group and standard care group respectively and both differences were not significant statistically.

Another study showed that KMC infants gain larger daily weight, while they were cared in hospital (control: $10.4 \pm$ 4.8 grams, KMC $19.3 \pm 3.8$ grams, $p<0.001) .{ }^{7}$ In a meta-analysis by Neonatal Review Group of the Cochrane Collaboration randomized trials comparing KMC and conventional neonatal care in LBW showed KMC neonates had gained more weight per day by discharge than controls (weighted mean difference $3.6 \mathrm{~g} /$ day, $95 \%$ confidence interval 0.8 to 6.4$){ }^{32}$

\section{CONCLUSIONS}

Kangaroo Mother Care maintain effective thermal control, decrease the incidence of sepsis, reduces feed intolerance, helps to achieving full enteral feeding and birth weight earlier in preterm low birth weight neonates. KMC also found to reduce hospital stay, treatment cost and enhances growth during postnatal period.

\section{RECOMMENDATION}

With proper implementation, KMC may become a safe and effective method in management of preterm low birth weight babies. 


\section{REFERENCES}

1. Lawn JE, Cousens S, Zupan J. 4 million neonatal deaths: when? Where? Why? Lancet. 2005; 365: 891-90

2. Blencowe H, Cousens SO, Estergaard M, Chou D, Moller AB, Adler A, et al. National regional and worldwide estimates of preterm birth rates in the year 2010 with time trends since 1990 for selected countries :a systematic analysis and implications. The Lancet. 2012; 9(379):2162-72.

3. Bangladesh Demographic and Health Survey Preliminary report, 2011

4. Gomella, TL.Temperature Regulation. Neonatology: Management, procedures, On-call Problems, Diseases, and Drugs. Sixth edition. New york: McGraw- Hill; 2009. pp. 43-47

5. Rey E, Martinez-H, Manejoracinal del nino premature. Bogota, Colombia: Universadad National, Curso de Medicina Fetal, 1983

6. Charpak N, Ruiz-Pelaez JG, Figueroa de CZ, Charpak Y. Kangaroo mother versus traditional care for newborn infants $</=2000$ grams: a randomized, controlled trial. Pediatrics.1997; 100(4):682-8.

7. Ali SM, Sharma J, Sharma R., Alam S. Kangaroo mother care as compared to conventional care for low birth weight babies. Dicle Med J cilt.2009; 36: 155-60.

8. Drosten-Brooks F. Kangaroo care: Skin-to-skin contact in the NICU. MCN.American Journal of Maternal Child Nursing. 1993; 18(5): 250-53.

9. Sloan NL, Camacho LW, Rojas EP, Stern C. Kangaroo mother method: randomized controlled trial of an alternative method of care for stabilized low- birthweight infants. Lancet.1994; 344(8925): 782-5.

10. Wahlberg V, Affonso D, Persson B . A retrospective, comparative study using the kangaroo method as a complement to standard incubator care. European Journal of Public Health.1992; 2(1): 34-37

11. Affonso D, Wahelberg B, Persson B. Exploration of mothers reactions to the kangaroo method of prematurity care. Neonatal Network. 1989; 7(6):43-51

12. Johnson LL, Cousen HL, Lawn JE.Global, regional and national causes of child mortality in 2000-2010: an update systematic analysis. The Lancet, June 2012; doi:10.1016/SO140-6736(12)60560-1.

13. Rao PN, Udani R., Nanavati R. Kangaroo mother care for low birth weight infants: a randomized controlled trial. Indian Pediatric.2008; 45(1):17-23.

14. Feldman R., EidelmanAI.Intervention Programs for premature infants. ClinPerinatol.1998; 25: 613-26.

15. Bauer K, Uhrig C, Sperling P, Pasel K, Wieland C, Versmold H T.Body temperatures and oxygen consumption during skin-to-skin (kangaroo) care in stable preterm infants weighing less than 1500 grams. 1997. J Pediatr. 1997; 130: 240-44.

16. Ludington-Hoe SM, Hadeed AJ, Anderson GC. 1991). Physiologic responses to skin- to-skin contact in hospitalized premature infants.JPerinato.1991; 11:19-24.

17. IbeOE, Austin T, Sullivan K, Fabanwo O, Disu E, Costello AM. A comparison of KMC and conventional Incubator care for thermal regulation of infants $<2000 \mathrm{~g}$ in Nigeria using continuous ambulatory temperature monitoring.Ann Trop Pediatr. 2004; 24:245- 51.

18. Cattaneo A, Danavzo R, Bergman NJ, Charpak N.Kagaroo mother care in low- income countries. J Trop Pediatr.1998; 44:279-82.

19. Conde-Agudelo A, Diaz-Rossello JL, Belizan JM. Kangaroo mother care to reduce morbidity and mortality in low birth weight infants. Cochrane Database Syst Rev. 2003;2:CD002771.

20. Subedi K, Aryal DR, Gurubacharya SM. Kangaroo Mother Care for Low Birth Weight Babies: A prospective Observational Study. J Nepal Pediatric. Soc. 2009; 29(1): 6-9.

21. Sloan NL, Camacho LW, Rojas EP, Stern C. Kangaroo mother method: randomized controlled trial of an alternative method of care for stabilized low- birth weight infants. Lancet.1994; 344: 782-85.

22. Kadam S, Binoy S, Kanbur W.Feasibility of kangaroo mother care in Mumbai.Indian J Pediatr.2005; 72:35-38.

23. Ramanathan. KPaul VK, Deorari AK, Taneja U, George G.Kangaroo Mother Care in very low birth weight infants.Indian J Pediatr.2001; 68(11): 1019-23.

24. Renfrew MJ, Craig D, Dyson L.Breastfeeding promotion for infants in neonatal units:A systematic review and economic analysis.HealthTechnol Assess.2009; 13(40):151-9

25. Hake-Brooks J, AndersonGC. Kangaroo care and breastfeeding of mother-preterm infant dyads $0-18$ 
months: A randomized controlled trial. Neonatal Netw.2008; 27(3):151-9.

26. American Academy of Pediatrics. Workgroup on breastfeeding and use of human milk.Pediatrics.1997; 100:1035-9

27. Amin SB, Merle KS, Orlando MS, Dalzell LE, Guillet R. Brain stem maturation in premature infants as a function of enteral feeding type. Pediatrics. 2001;106:318-20.

28. Hurst NM, Valentine CJ, Renfro L, Burns P, Ferlic L. Skin-to-skin holding in the neonatal intensive care unit influences maternal milk volume. J Perinatol.1997; 17(3):213-7.

29. Whitelaw A, Heiterkamp G, Sleath K, Acolet D, Richards M. Skin to skin contact for very low birth weight infants and theirs. Arch Dis Child. 1988; 63(11): 1377-81.
30. Ramanathan K, Paul VK, Deorari AK, Taneja U, GeorgeG.Kangaroo Mother Care in very low birth weight infants. Indian J Pediatr. 2001; 68(11): 1019-23.

31. Conde-Agudelo A, Belizan JM., Diaz-Rossello J. Kangaroo mother care to reduce morbidity and mortality in low birthweight infants, BMC Pregnancy Childbirth. Cochrane Database of Syst Rev. 2011; 3: CD002771

32. Ibe OE, Austin T, Sullivan K, Fabanwo O, Disu E, Costello AM. A comparison of KMC and conventional Incubator care for thermal regulation of infants $<2000 \mathrm{~g}$ in Nigeria using continuous ambulatory temperature monitoring. Ann Trop Pediatr. 2004; 24:245- 51. 